\title{
Mutation spectrum of the FZD-4, TSPAN12 AND ZNF408 genes in Indian FEVR patients
}

\author{
Ganeswara Rao Musada', Hameed Syed', Subhadra Jalali², Subhabrata Chakrabarti ${ }^{1}$ and Inderjeet Kaur ${ }^{\text {* }}$
}

\begin{abstract}
Background: Mutations in candidate genes that encode for a ligand (NDP) and receptor complex (FZD4, LRP5 and TSPAN12) in the Norrin $\beta$-catenin signaling pathway are involved in the pathogenesis of familial exudative vitreoretinopathy (FEVR, MIM \# 133780). Recently, a transcription factor (ZNF408) has also been implicated in FEVR. We had earlier characterized the variations in NDP among FEVR patients from India. The present study aimed at understanding the involvement of the remaining genes (FZD4, TSPAN12 and ZNF408) in the same cohort.

Methods: The DNA of 110 unrelated FEVR patients and 115 unaffected controls were screened for variations in the entire coding and untranslated regions of these 3 genes by resequencing. Segregation of the disease-associated variants was assessed in the family members of the probands. The effect of the observed missense changes were further analyzed by SIFT and PolyPhen-2 scores.

Results: The screening of FZD4, TSPAN12 and ZNF408 genes identified 11 different mutations in 15/110 FEVR probands. Of the 11 identified mutations, 6 mutations were novel. The detected missense mutations were mainly located in the domains which are functionally crucial for the formation of ligand-receptor complex and as they replaced evolutionarily highly conserved amino acids with a SIFT score $<0.005$, they are predicted to be pathogenic. Additionally 2 novel and 16 reported single nucleotide polymorphisms (SNP) were also detected.

Conclusions: Our genetic screening revealed varying mutation frequencies in the FZD4 (8.0 \%), TSPAN12 (5.4 \%) and ZNF408 (2.7 \%) genes among the FEVR patients, indicating their potential role in the disease pathogenesis. The observed mutations segregated with the disease phenotype and exhibited variable expressivity. The mutations in FZD4 and TSPAN12 were involved in autosomal dominant and autosomal recessive families and further validates the involvement of these gene in FEVR development.
\end{abstract}

Keywords: Retina, Abnormal angiogenesis, Mutation screening, Candidate genes

\section{Background}

FEVR is a potentially blinding eye disorder caused by mutations in genes that play a crucial role in the development of the normal retinal vasculature [1-10]. It is primarily characterized by a failed development of the peripheral retinal vasculature on the temporal side of the retina [11-13]. This feature is asymptomatic and observed in most patients without any further progression during their life time $[7,11,12]$. However, it might lead

\footnotetext{
* Correspondence: inderjeet@lvpei.org; ikaurs@gmail.com

${ }^{1}$ Kallam Anji Reddy Molecular Genetics Laboratory, Brien Holden Eye

Research Centre, L V Prasad Eye Institute (KAR Campus), Road\#2, Banjara Hills, Hyderabad 500034, India

Full list of author information is available at the end of the article
}

to secondary clinical complications including neovascularization, retinal traction with ectopic macula, exudation, subtotal and total retinal detachment in some patients $[7,11,12]$. The age of onset in FEVR is variable but the severe disease phenotype is usually observed in the first or second decade of a patient's life. A highly variable expressivity between the two eyes of the same patient and among the different family members is another commonly reported feature of this disease $[7,11,12]$.

So far, mutations in 6 candidate genes have been attributed to the development of FEVR including Norrie disease pseudoglioma (NDP; OMIM 300658) [1, 9, 10, 14, 15], Frizzled-4 (FZD4; OMIM 604579) [1, 2, 7, 8, 14-20], Low 
density lipoprotein receptor like protein 5 (LRP5; OMIM 603576) [1, 2, 6, 14-16, 19, 21], Tetraspanin-12 (TSPAN12; OMIM 613138) [1, 2, 4, 5, 22-25], Zinc finger prortein408 (ZNF408) [1,3] and Kinesin family member 11 (KIF11) [26, 27]. Mutations in these genes have resulted in autosomal dominant (FZD4, LRP5, TSPAN12, ZNF408 and $K I F 11)[[3,7,8,14-16,24,26,27]$, autosomal recessive (LRP5, TSPAN12) [21, 23] and X-linked (NDP) FEVR $[9,10]$ and have accounted for $\sim 50 \%$ of all patients [1-3]. Among these, the autosomal dominant mode of inheritance has been widely reported. The proteins encoded by FEVR candidate genes (excluding ZNF408 and KIF11) have been shown to be involved in the formation of a signal transduction complex of Norrin$\beta$ catenin signaling pathway [28-32]. The knockout mouse models of these genes have exhibited phenotype resembling FEVR and have provided further evidence on the role of this signaling pathway in the formation, maturation and maintenance of intraretinal vasculature [28-31, 33-37]. Norrin, a protein encoded by NDP gene being similar to canonical-Wnt signaling pathway, acts as a ligand and interacts with FZD4-LRP5 receptor complex. This interaction further stabilizes the cytoplasmic $\beta$ catenin molecules to induce the T-cell factor/lymphoid enhancer factor (TCF/LEF) mediated gene expression after its translocation into the nucleus [30-32]. TSPAN12 enhances the Norrin signaling by augmentation of FZD4 multimerization [28]. However, the roles of the ZNF408 and KIF11 genes in the developmental retinal angiogenesis and Norrin- $\beta$ catenin signaling pathway is yet unknown.

The FZD4 is a 537 amino acid protein containing an Nterminal extracellular domain (composed of signal sequence, FZ domain and a linker region), 7 transmembrane hydrophobic helical domains, 3 extracellular, 3 intracellular loops and a $C$ terminal cytoplasmic domain $[38,39]$. The extracellular FZ domain is cysteine rich (CRD) and is essential for the recognition and binding to FZD4 specific ligands [40, 41]. The cytoplasmic $C$ terminal region contains two highly conserved PDZ binding domains (postsynaptic density 95/disc-large/zona occludens-1: KTXXXW and ETVV) and play a crucial role in recruiting downstream regulator proteins to induce the signaling mechanism [39]. The TSPAN12 gene is located on chromosome 7q31 and encodes for a 305 amino acid transmembrane protein. Structurally, this protein contains four transmembrane domains connected by two extra cellular loops (ECL-1 and 2) and an intra cellular loop, and intracellular $\mathrm{N}$ and $\mathrm{C}$ terminals. The ECL- 1 is small compared to the ECL- $2[4,5,22]$.

Recently, Collin et al. [3] functionally characterized this new candidate gene ZNF408 in FEVR. A novel founder mutation was observed in two Dutch families and further screening of 132 patients revealed another mutation in a Japanese patient. The ZNF408 gene encodes for a 720 amino acid protein and predicted to contain ten zinc finger
DNA binding domains. Based on the presence of zinc finger domains, this protein was suggested to be a transcription factor [3]. Functional analysis of mutant proteins indicated a dominant negative disease mechanism and subsequent knockdown of Znf408 in zebrafish, suggested its putative role in retinal vasculogenesis.

So far, 88 different mutations have been reported in FZD4 $(n=58)$, TSPAN12 $(n=27)$ and ZNF408 $(n=3)$ in patients with FEVR and other vitreo-retinal abnormalities. These genes have been quite well characterized in Caucasian, Chinese and Japanese FEVR patients. However, in the Indian context, there is only a single report from a relatively smaller cohort of FEVR patients on FZD4 gene screening [42] (Nallathambi J et al.,). Thus, the present study was undertaken to explore the involvement of the FZD4, TSPAN12 and ZNF408 genes and to assess their mutation spectrum in a large cohort of Indian FEVR patients.

\section{Methods}

The study was approved by the Institutional Review Board (IRB) of L.V. Prasad Eye Institute, Hyderabad, India, (Ref no LEC06104) and adhered to tenets of the Declaration of Helsinki. We recruited 110 unrelated FEVR cases (34 familial and 76 sporadic) along with their family members after obtaining a written informed consent. All the patients had a history of full term birth. A senior ophthalmologist examined all the patients and diagnosed the disease based on indirect ophthalmoscopic examination, B-scan ultrasonography and fundus fluorescein angiography in selective patients. As described previously, the diagnosis of FEVR was confirmed based on the presence of bilateral peripheral retinal avascularity along with or without any of the ocular features such as subretinal exudation, neovascularization, vitreoretinal traction, retinal folds, ectopic macula and partial or total retinal detachments [7, 12, 20]. The different stages of the disease were ascertained based on the Pendergast and Trese classification [12]. The diagnosis and staging of the disease was independently confirmed by another ophthalmologist through evaluation of the medical records and fundus photographs of the patients. Additionally, 115 ethnically matched normal subjects aged over 60 years and without any present and past history of retinal disorders nor signs or symptoms of FEVR or any other ocular or systemic conditions were enrolled as controls. They were generally subjects with senile cataract who were also evaluated for any other ocular complications through a comprehensive eye examination.

\section{Mutation screening of the FZD4 and TSPAN12 genes}

Genomic DNA was extracted from peripheral blood leucocytes using standard protocols of Phenol-Chloroform extraction method [43]. Mutation screening of entire coding and intron-exon boundaries of FZD4, TSPAN12 and ZNF408 genes were accomplished by polymerase 
chain reaction (PCR) based amplification of these regions with 26 different sets of overlapping primers followed by resequencing on an automated DNA sequencer ABI 3130 XL (Applied Biosystems, Foster City, CA) using the Big Dye chemistry (version 3.1) following the manufacturer's guidelines. The primers used for PCR and their optimum annealing temperatures are listed in Additional file 1: Table S1. The observed variations were further validated by resequencing. Samples of the available family members of the respective probands in the FEVR-affected families were also screened for assessing the segregation of the identified variations. In addition to the screening of the observed variants in the ethnically matched controls, their allele frequencies were also checked in the dbSNP [44], ESP5400 [45], NIEHS95 [46] and ExAC [47] databases. All the identified nonsynonymous substitutions were assessed to predict their functional significance by computational prediction algorithims; SIFT [48], PolyPhen-2 [49], and Mutation Taster [50]. Multiple sequence alignments were carried out using Clustal W to determine the evolutionary conservation of wild type amino acid residues at the position of substitutions [51]. Evolutionarily non-conservative missense changes predicted as deleterious by at least two computational tools were considered as potentially pathogenic mutations. The remaining missense changes were categorized as variants of unknown significance.

\section{Results}

The screening of the entire coding and flanking regions of FZD4, TSPAN12 and ZNF408 genes revealed a total of 11 potentially pathogenic mutations in 15 different FEVR probands (Table 1 and Fig. 1). Of these 11 mutations, six were novel. The novel mutations included a missense change (c.341 T > G; p.Ile114Ser), a single base pair insertion (c.1395_1396insT; p.Arg466Serfs*6) and a non-stop change (c.1613A > C; p.*538Serext*2) in the FZD4 gene. Three novel mutations c.125 T>C (p.Val42Ala), c.479G > A (p.Cys160Tyr) and c.2145G > T (p. Glu715Asp) were also observed in the TSPAN12 and ZNF408, respectively (Table 1 and Fig. 1). In addition to these novel changes, two previously reported missense mutations; c.313A > G; [p.Met105Val] $[1,2,16,18,20]$ and c.470 T > C [p.Met157Thr] [2], two small base pair deletions; c.1282-1285delGACA [p.Asp428Serfs"2] [1, 2, 15, 16] and c.1286-1290delAGTTA [p.Lys429Argfs*28] [52] in the FZD4 gene; a rare variant in TSPAN12 gene; c.334G > A [p.Val112Ile] and two novel missense changes (c.130C > T [p.Pro44Ser] and c.694A > G [p.Met232Val]) in ZNF408 of unknown significance were also observed. None of these mutations were observed in the 115 normal controls. Apart from these mutations, three reported single nucleotide polymorphisms (SNPs); c.502C > T [rs61735303], c.576C $>\mathrm{T}$ [rs2011686860] and c.1078 A > G [rs530613772] were observed in FZD4 gene.
Two intronic (IVS2 + 24C > G [rs545602315] and IVS6 + 19G > C) and five 3' UTR single nucleotide substitu-

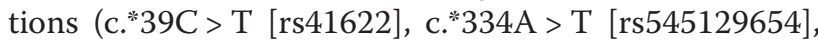
c." $1010 \mathrm{~T}>\mathrm{G}, \mathrm{c.}{ }^{*} 1140 \mathrm{~T}>\mathrm{A}$ and $\mathrm{c}^{*} 1243 \mathrm{~A}>\mathrm{T}$ [rs1892 21112]) were observed in the TSPAN12 gene. Likewise, two novel 5' UTR variations (c. -214_-210delGAATC and $-111 \mathrm{C}>\mathrm{A}$ ) and six SNPs (c.402A $>\mathrm{G}$ [rs5613 20549], c.408C > A, c.576_587del12bp [rs148055528], c.581_592del12bp [rs72461400] c.1971A > G [rs37653 6252] c. $1850 \mathrm{C}>\mathrm{A}$ [rs547169524] were observed in ZNF408.

All the nucleotide changes observed in FZD4 gene were heterozygous. Excluding the c.313A > G (p.Met105Val) and c.1286-1290delAGTTA (p.Lys429Argfs*28) mutations, the remaining mutations were observed in one proband each. A missense p.Met105Val mutation was observed in three different probands and likewise a frameshift p.Lys429Argfs $* 28$ mutation was observed in two different probands. Interestingly, a FEVR proband harbored two changes: c.1282-1285delGACA (p.Asp428Serfs*2) and c.1613A > C (p."538Serext"2) in the FZD4 gene.

In TSPAN12, two mutations c.125 $\mathrm{T}>\mathrm{C}$ (p.Val42Ala) and c.334G > A (p.Val112Ile) were identified in one proband each, while the c.479G > A (p.Cys160Tyr) mutation was observed in four different probands, wherein, two of them harbored heterozygous changes and the remaining were homozygous. The p.Val112Ile mutation has also been identified in homozygous condition whereas, p.Val42Ala mutation was observed in heterozygous condition. The wild type valine residue at codon 42 was highly conserved across different species. However, this change was predicted to be non-pathogenic by PolyPhen- 2 whereas, $\mathrm{Mu}-$ tation Taster and SIFT indicated for the harmful effect of this substitution.

Three heterozygous missense changes (p.Pro44Ser, p.Met232Val and p. Glu715Asp) identified in ZNF408, were observed in one proband each. Of the total nine missense changes identified in the present study, except p.Pro44Ser and p.Met232Val changes of ZNF408, the remaining were predicted as deleterious by at least two computational prediction tools used for in silico analysis. Furthermore, the wild type amino acids located at these missense changes were highly conserved across different species (Fig. 2). Thus, these missense changes were categorized as potentially pathogenic mutations, while the p.Pro44Ser and p.Met232Val changes of ZNF408 were variations of unknown significance.

\section{Discussion}

In the present study, several novel mutations (missense, non-stop and insertion) were detected in the coding regions of FZD4, TSPAN12 and ZNF408 genes among the unrelated FEVR probands. None of these nucleotide changes were observed in 115 ethnically matched controls 
Table 1 Mutations observed in FZD4, TSPAN12 and ZNF408 genes in FEVR patients

\begin{tabular}{|c|c|c|c|c|c|c|c|c|c|c|}
\hline \multirow{2}{*}{$\begin{array}{l}\text { Family } \\
\text { number }\end{array}$} & \multirow{2}{*}{ Gene/Exon } & \multirow{2}{*}{$\begin{array}{l}\text { CDNA } \\
\text { change }^{a}\end{array}$} & \multirow{2}{*}{$\begin{array}{l}\text { Protein } \\
\text { change }^{b}\end{array}$} & \multicolumn{3}{|c|}{ In silico analysis } & \multicolumn{2}{|c|}{ Occurrence in } & \multirow{2}{*}{$\begin{array}{l}\text { Novel/ } \\
\text { Reported }\end{array}$} & \multirow{2}{*}{$\begin{array}{l}\text { Functional } \\
\text { significance }\end{array}$} \\
\hline & & & & $\begin{array}{l}\text { SIFT } \\
\text { (score) }\end{array}$ & $\begin{array}{l}\text { PolyPhen-2 } \\
\text { (score) }\end{array}$ & $\begin{array}{l}\text { Mutation Taster } \\
\text { (probability value) }\end{array}$ & Patients & Controls & & \\
\hline 1 & $F Z D 4 / 2$ & c.341T>G & p.lle114Ser & $\begin{array}{c}\text { Damaging } \\
(0.00)\end{array}$ & $\begin{array}{l}\text { Probably } \\
\text { damaging } \\
(0.996)\end{array}$ & $\begin{array}{c}\text { Disease causing } \\
(0.9999)\end{array}$ & $1 / 110$ & $0 / 115$ & Novel & Pathogenic \\
\hline 2 & $F Z D 4 / 2$ & $\begin{array}{l}\text { c.1395 } \\
\text { 1396insT }\end{array}$ & $\begin{array}{l}\text { p.Arg466Ser } \\
\text { fs*6 }\end{array}$ & - & - & - & $1 / 110$ & $0 / 115$ & Novel & Pathogenic \\
\hline \multirow[t]{2}{*}{3} & \multirow[t]{2}{*}{$F Z D 4 / 2$} & C. $1613 A>C$ & p. $* 538$ Serext*2 & - & - & - & $1 / 110$ & $0 / 115$ & Novel & Pathogenic \\
\hline & & $\begin{array}{l}\text { c.1286-1290 } \\
\text { delAGTTA }\end{array}$ & $\begin{array}{l}\text { p.Lys } 429 \operatorname{Arg} \\
\mathrm{fs}^{*} 28\end{array}$ & - & - & - & $1 / 110$ & $0 / 115$ & Reported [52] & Pathogenic \\
\hline $\begin{array}{l}4 \\
5\end{array}$ & $F Z D 4 / 2$ & $\begin{array}{l}\text { c.1282_1285 } \\
\text { delGACA }\end{array}$ & $\begin{array}{l}\text { p.Asp428Ser } \\
\mathrm{fs}^{*} 2\end{array}$ & - & - & - & $2 / 110$ & $0 / 115$ & $\begin{array}{c}\text { Reported } \\
{[1,2,15,16]}\end{array}$ & Pathogenic \\
\hline \multirow[t]{2}{*}{6} & $F Z D 4 / 2$ & c. $470 \mathrm{~T}>\mathrm{C}$ & p.Met157Thr & $\begin{array}{l}\text { Damaging } \\
(0.039)\end{array}$ & $\begin{array}{l}\text { Benign } \\
(0.256)\end{array}$ & $\begin{array}{c}\text { Disease causing } \\
(0.9999)\end{array}$ & $1 / 110$ & 0/115 & Reported [2] & Pathogenic \\
\hline & ZNF408/5 & c. $694 \mathrm{~A}>\mathrm{G}$ & p.Met232Val & $\begin{array}{c}\text { Tolerated } \\
(0.508)\end{array}$ & $\begin{array}{l}\text { Benign } \\
(0.000)\end{array}$ & $\begin{array}{l}\text { Polymorphism } \\
\text { (0.9999) }\end{array}$ & $1 / 110$ & $0 / 115$ & Novel & $\begin{array}{l}\text { Unknown } \\
\text { significance }\end{array}$ \\
\hline 7 & \multirow[t]{3}{*}{$F Z D 4 / 2$} & \multirow[t]{3}{*}{ c. $313 A>G$} & \multirow[t]{3}{*}{ p.Met105Val } & \multirow{3}{*}{$\begin{array}{c}\text { Tolerated } \\
(0.858)\end{array}$} & \multirow{3}{*}{$\begin{array}{c}\text { Possibly } \\
\text { damaging } \\
(0.793)\end{array}$} & \multirow{3}{*}{$\begin{array}{l}\text { Disease causing } \\
\quad(0.9999)\end{array}$} & \multirow[t]{3}{*}{$3 / 110$} & \multirow[t]{3}{*}{$0 / 115$} & \multirow{3}{*}{$\begin{array}{c}\text { Reported } \\
{[1,2,16,18,20]}\end{array}$} & \multirow[t]{3}{*}{ Pathogenic } \\
\hline 8 & & & & & & & & & & \\
\hline 9 & & & & & & & & & & \\
\hline 10 & TSPAN12/3 & c. $125 \mathrm{~T}>\mathrm{C}$ & p.Val42Ala & $\begin{array}{l}\text { Damaging } \\
(0.023)\end{array}$ & $\begin{array}{l}\text { Benign } \\
(0.127)\end{array}$ & $\begin{array}{c}\text { Disease causing } \\
(0.9998)\end{array}$ & $1 / 110$ & $0 / 115$ & Novel & Pathogenic \\
\hline 11 & \multirow[t]{4}{*}{ TSPAN12/7 } & \multirow[t]{4}{*}{ c. $479 \mathrm{G}>\mathrm{A}$} & \multirow[t]{4}{*}{ p.Cys160Tyr } & \multirow{4}{*}{$\begin{array}{l}\text { Damaging } \\
(0.000)\end{array}$} & Probably & Disease causing & $4 / 110$ & $0 / 115$ & Novel & Pathogenic \\
\hline 12 & & & & & $\begin{array}{c}\text { damaging } \\
(1.000)\end{array}$ & & & & & \\
\hline 13 & & & & & & & & & & \\
\hline 14 & & & & & & & & & & \\
\hline 15 & TSPAN12/5 & c.334G $>A$ & p.Val112lle & $\begin{array}{c}\text { Tolerated } \\
(0.338)\end{array}$ & $\begin{array}{l}\text { Possibly } \\
\text { damaging } \\
(0.533)\end{array}$ & $\begin{array}{c}\text { Disease causing } \\
(0.9999)\end{array}$ & $1 / 110$ & $0 / 115$ & Reported [47] & Pathogenic \\
\hline 16 & ZNF408/2 & c. $130 C>T$ & p.Pro44Ser & $\begin{array}{c}\text { Tolerated } \\
(0.054)\end{array}$ & $\begin{array}{l}\text { Benign } \\
(0.062)\end{array}$ & $\begin{array}{l}\text { Polymorphism } \\
\text { (0.9999) }\end{array}$ & $1 / 110$ & $0 / 115$ & Novel & $\begin{array}{l}\text { Unknown } \\
\text { significance }\end{array}$ \\
\hline 17 & ZNF408/5 & $c .2145 G>T$ & p.Glu715Asp & $\begin{array}{l}\text { Damaging } \\
(0.003)\end{array}$ & $\begin{array}{c}\text { Probably } \\
\text { damaging } \\
(0.966)\end{array}$ & $\begin{array}{c}\text { Disease causing } \\
\quad(0.888)\end{array}$ & $1 / 110$ & $0 / 115$ & Novel & Pathogenic \\
\hline
\end{tabular}

${ }^{a}$ NCBI Reference Sequences for FZD4, TSPAN12 and ZNF408 mRNA are NM_012193.2, NM_012338.3 and NM_024741.2 respectively. ${ }^{b}$ NCBI Reference Sequences for FZD4, TSPAN12 and ZNF408 proteins are NM_036325.2, NP_036470.1 and NP_079017.1 respectively. The effect of missense changes were predicted using SIFT, PolyPhen-2 and Mutation Taster online prediction tools. In SIFT, the scores less than 0.05 were considered as damaging. In PolyPhen-2, the scores less than 0.5 were considered as disease causing

and were not present in the dbSNP [44], ESP5400 [45], NIEHS95 [46] and ExAC [47] databases. The probands harboring the mutations in these genes exhibited severe disease phenotype with neovascularization, falciform retinal folds, exudative or tractional retinal detachments and severe visual impairment. Some of these mutations were also observed in the family members of the probands who exhibited a highly variable phenotype based on indirect ophthalmoscopic examination. The phenotype of the family members ranged from normal retinal vascularization or very mild avascularization to neovascularization, partial and/or total retinal detachments with or without exudation. Since, some of the asymptomatic family members with the identified mutations were unavailable for fluorescein fundus angiography, we could not rule out the possibility of missing very mild retinal vascular changes. The patients identified with FZD4 or TSPAN12 or ZNF408 mutations exhibited classical clinical features of FEVR, but failed to exhibit any genotype-phenotype correlation.

\section{Mutations detected in the FZD4 gene}

A total of 58 different mutations have been reported in FZD4 in FEVR patients, including 30 missense mutations, 10 deletions, 9 nonsense mutations and two singe bp insertions $[1,2,7,8,14-20,42,51-55]$. A heterozygous novel c.1395_1396insT (p.Arg466Serfs*6) change resulting in a frame shift at codon 466 leading to a premature termination codon, was observed in a familial case of 


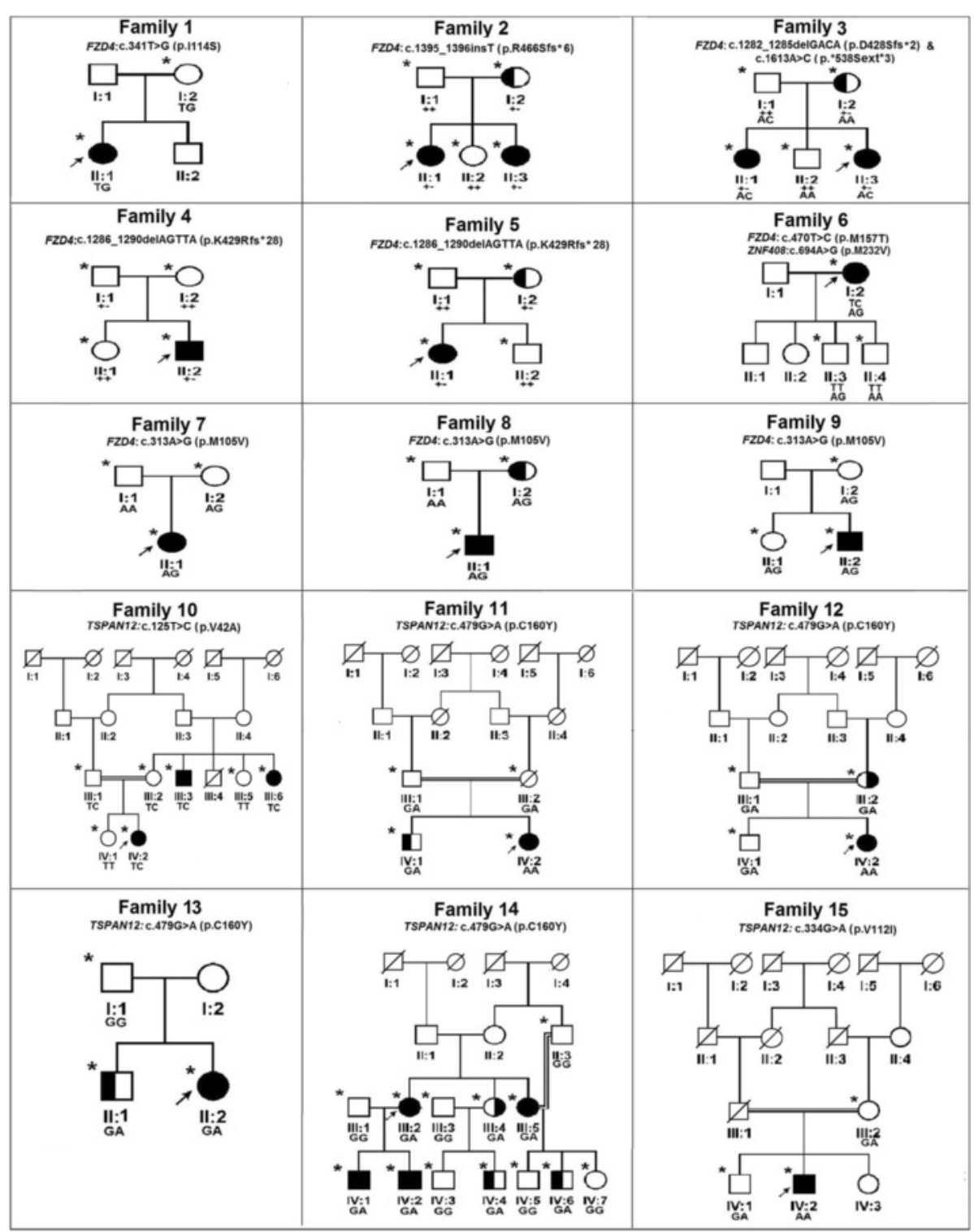

Fig. 1 Pedigrees of the FEVR families with nucleotide changes in FZD4 and TSPAN12 genes. Completely shaded symbols represent severe stages of the disease. Open symbols represent unaffected individuals. Asterisk $\left(^{*}\right)$ over the pedigree symbols represent the individuals screened for nucleotide changes in FZD4 and TSPAN12 genes. - indicates the presence of deletion/insertion and + indicates the wild type allele. The identified nucleotide change is represented above each pedigree

FEVR (Fig. 1; family 2; II:1) that segregated in all the affected family members. The proband and her female siblings (II:1 and II:3) with this frameshift mutation developed bilateral vitreo retinal traction and total retinal detachment at their infancy while their mother (I:2) who was a carrier for this mutation, had avascularized peripheral retina (Fig. 3). This is the third insertion mutation to be observed in the FZD4 gene so far.

A novel non-stop change (c.1613A > C; p."538Serext"2) along with a previously reported 4 base pair deletion (c.1282-1285delGACA; p.Asp428Serfs*2) [1, 2, 15, 16] was observed in a familial case in a compound heterozygous condition. This change resulted in disruption of proper termination and addition of two extra amino acids to the wild type protein. A severe disease phenotype with bilateral total retinal detachments was observed in the proband (II:3) and her female sibling (II:1) that might have resulted from the synergistic effect of both the mutated alleles. The father (I:1) and mother (I:2) of these siblings were carriers for the mutated alleles c.1613A > C and c.1282-1285delGACA, respectively The parents of these siblings harboring either of the mutated alleles were normal or exhibited avascularized peripheral retina. The nonstop mutation has been identified for the first time in 


FZD4
Homo sapiens
Macaca mulatta
Rattus norvegicus
Mus musculus
Gallus gallus
Xenopus laevis
Canis lupus familiaris
Equus caballus
Elephantulus edwardii
Alligator mississippiensis
Bos taurus
Lipotes vexilifer
Sus scrofa

TSPAN12

Homo sapiens
Macaca mulatta
Rattus norvegicus
Mus musculus
Gallus gallus
Bos taurus
Python bivittatus
Danio rerio
Otolemur garnetti
Lipotes vexillifer
Ovis aries
Erinaceus europaeus
Sarcophilus harrisii
ZNF408
Homo sapiens
Macaca mulatta
Felis catus
Canis lupus familiaris
Equus caballus
Pantroglodytes
Bostaurus
Callithrix jacchus
Condylura cristata
Sus scrofa
Elephantulus edwardii

\begin{tabular}{|c|c|}
\hline p.M105V & p.I114S \\
\hline 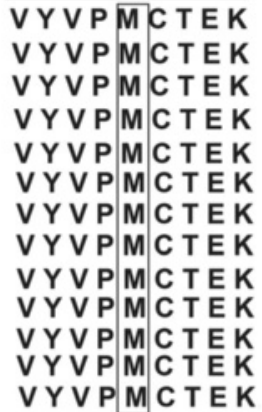 & 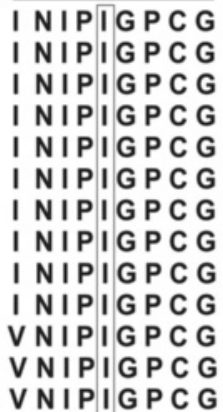 \\
\hline
\end{tabular}

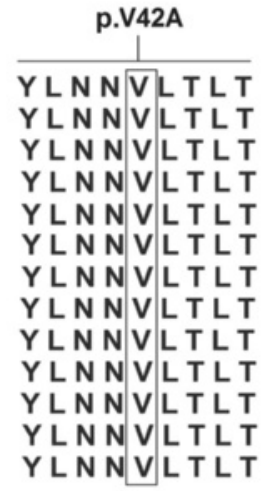

p.P44S

LKDV P P P T

LKD VPP G P T

LKD F P P G T

LKDFPPE P T

R R D F P P P T

LKD V P P K P T

LKD V P P G P T

LKD VPPR S

LE DFPP G P

PKDFPARPT

C K N F P L P D

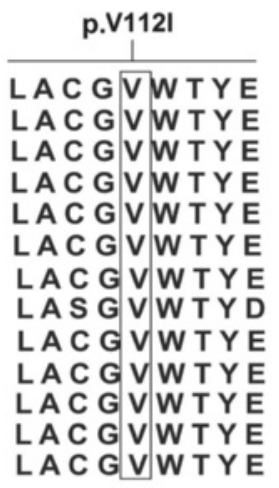

p.M232V

$\overline{\text { QAENMV S G }}$

QAESMVSPG

Q A E N ML S P G

QAESMVG PG

QAESMVS SG

Q A E N M VS P G

Q A E S V V S P G

QAASVVSPG

QAERVVSPE

QAESVVSS G

Q VKSPVSPG

\begin{tabular}{|c|}
\hline $\begin{array}{c}\text { p.M157T } \\
\end{array}$ \\
\hline 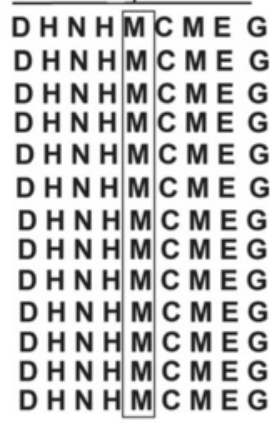 \\
\hline
\end{tabular}

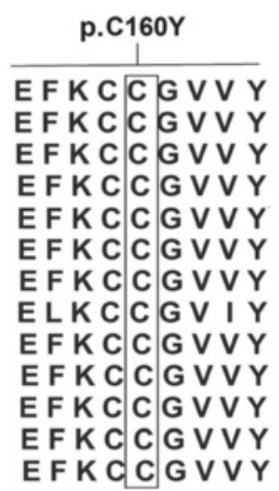

\begin{tabular}{|c|c|}
\hline & \\
\hline $\begin{array}{l}A E V V E \\
A E V V E \\
A E V V E \\
A E V V \\
A E V V E \\
A E V V E \\
A E V V \\
A E V V E \\
A E V V E \\
A E V V E\end{array}$ & $\begin{array}{l}E V E M G \\
\text { EVEMG } \\
\text { EVETG } \\
\text { EVETG } \\
\text { EVET G } \\
\text { EVE MG } \\
\text { EVET T } \\
\text { EVETG } \\
\text { EVET G } \\
\text { EVETG } \\
\text { VVET G }\end{array}$ \\
\hline
\end{tabular}

Fig. 2 Multiple sequence alignment showing the conservation of wild type residues in respect to identified missense changes in FZD4 protein, TSPAN12, and ZNF408 across various species. Except p.M232V of ZNF408 remaining all the missense mutations were highly conserved across different species. Reference sequences of the FZD4 orthologues: Homo sapiens; AAR23924.1, Macaca mulatta; NP_001253804.1, Rattus norvegicus; NP_072145.1, Mus musculus; NP_032081.3, Gallus gallus; NP_989430.1, Xenopus laevis; NP_001083922.1, Canis lupus familiaris; XP_005633439.1, Equus caballus; XP_001489854.1, Elephantulus edwardii; XP_006895316.1, Alligator mississippiensis; XP_006261662.1, Bos taurus; NP_001193198.1, Lipotes vexillifer; XP_007448903.1, Sus scrofa; XP_005667267.1. Reference sequences of the ZNF408 orthologues: Homo sapiens; NP_079017.1, Macaca mulatta; XP_001111210.1, Felis catus; XP_003993266.1, Canis lupus familiaris; XP_003639743.3, Equus caballus; XP_001490832.1, Pantroglodytes; JAA35243.1 Bos taurus; NP_001180086.1, Callithrix jacchus; XP_009006283.1, Condylura cristata; XP_004683622.1, Sus scrofa; XP_013849819.1, Elephantulus edwardii; XP_006896777.1

FZD4 gene and predicted to result in addition of two extra amino acids to the C-terminal cytoplasmic domain of FZD4 protein. Heterozygous compound mutations have previously been reported in FZD4 gene and could explain the inter and intra familial variability of disease phenotype $[18,20]$. The c.1282-1285delGACA change led to an open reading frame alteration at codon 428 and formation of a new stop codon at position 430 .
The third frameshift heterozygous alteration c.12861290delAGTTA (p.Lys429Argfs*28) was observed in two unrelated probands in families 4 and 5 (Fig. 1; family 4; II:2 and family 5; II:1). Both the probands developed total retinal detachment in one eye during early childhood and the affected eyes developed subtotal retinal detachment excluding the fovea. The mother (I:2) of the proband 5 who was a carrier for this deletion exhibited peripheral retinal 

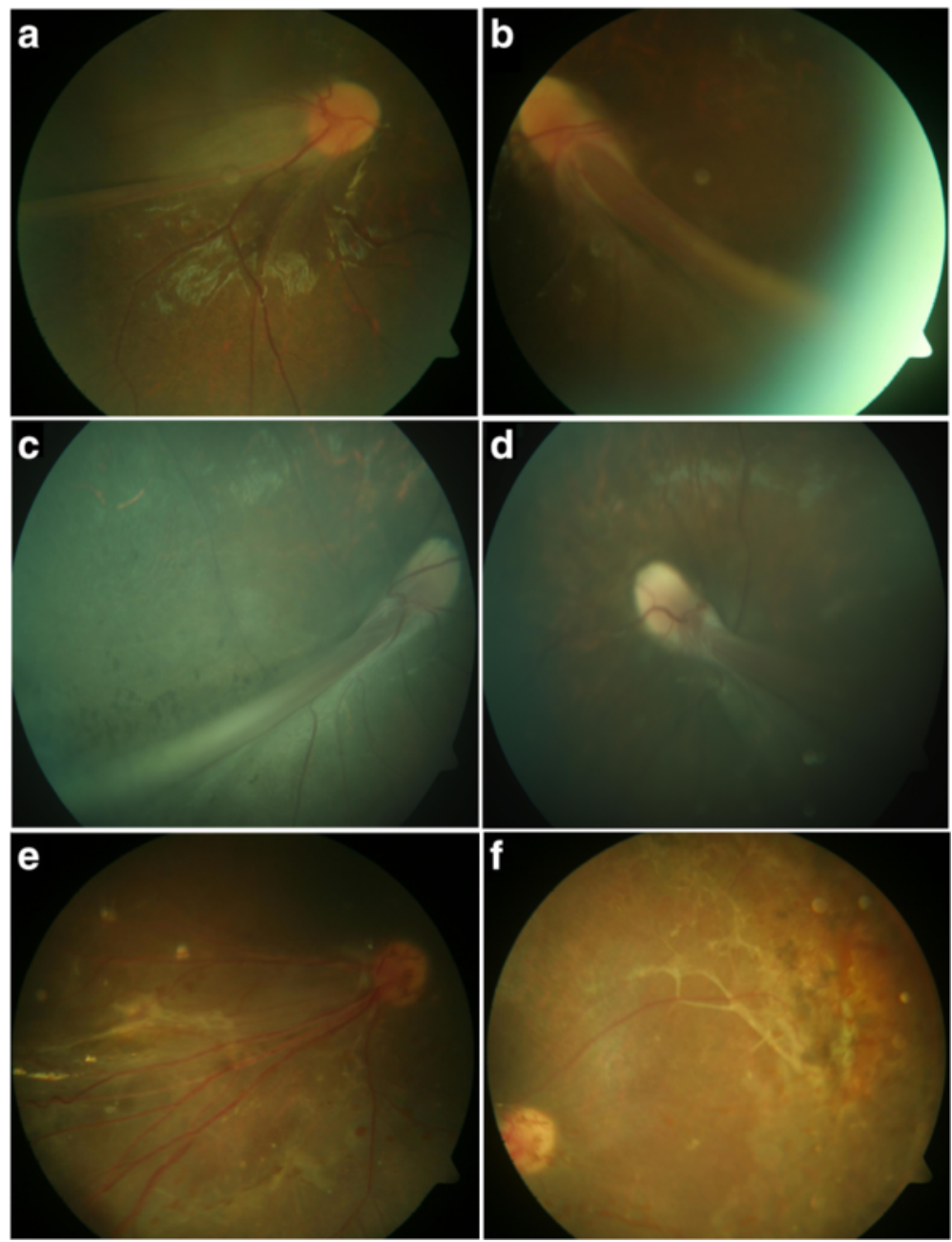

Fig. 3 Fundus photographs of the FEVR patients with novel nucleotide changes identified in the FZD4 gene. a\&b Patient ID: family 1-II:1 (c.341 T> G); Right and left eyes of proband of FEVR family 1 shows straightening of blood vessels and macular dragging toward inferotemporal area of the retina. c\&d Patient ID: family 2- II:1 (c.1395_1396insT); Right and left eyes of proband of FEVR family 2 shows macular dragging toward inferotemporal retina. e\&f Patient ID: family 3- II:3; Right and left eyes of proband of FEVR family 3 shows straightening of blood vessels, exudation and vitreoretinal traction

avascularization while the proband's father (I:1) despite having a single copy of the mutant allele did not exhibit any retinal vascular abnormality upon indirect ophthalmoscopy examination. This deletion led to frameshift at codon 429 along with the formation of a premature stop codon.

All the three frameshift mutations (p.Arg466Serfs $\%$, p.Asp428Serfs"2 and p.Lys429Argfs"28) were predicted to result in the formation of a truncated protein with a loss of 13 to $20 \%$ of the wild type FZD4 protein. Due to the presence of only two exons in FZD4, the frameshift mutations observed in the second exon may result in the formation of truncated protein rather than nonsense mediated mRNA decay [56]. The resulting truncated proteins affected the conserved KTXXXW domain and PDZ binding motif, essential for interaction with downstream regulator proteins. In the absence of these motifs, FZD4 would likely fail to induce the signaling pathway and consequently lead to haploinsufficiency. Alternatively, the truncated protein may oligomerize with wild type protein and become trapped in the endoplasmic reticulum as reported in the p.Lys501fs*533 mutation producing a dominant negative effect $[57,58]$.

Three missense mutations p.Met105Val, p.Ile114Ser and p.Met157Thr identified in the present study in FZD4 were located in the extracellular cysteine rich domain (CRD) of the gene. The wild type amino acid residues located at the 105, 114 and 157 codon positions were experimentally found to be crucial for ligand binding during Norrin signal transduction [30, 40, 41]. A novel heterozygous mutation, c.341 T > G (p.Ile114Ser) was identified in a female proband (II: 1 ) of family 1 (Fig. 1). The proband was noticed to have bilateral nystagmus with exudative retinal detachment including her 
macula at 8 months of age. The mother of the proband (I: 2) also harbored this change but was found to be clinically normal under indirect ophthalmoscopic examination (Fig. 3). Although, the p.Ile114Ser was identified in the present study, Robitaille et al., [53] reported a p.Ile114Thr mutation in a familial FEVR case with bilateral retinal folds at 2 months of age. The isoleucine residue at 114 codon position of FZD4 was highly conserved across multiple vertebrate species (Fig. 2). Replacement of hydrophobic isoleucine with a hydrophilic serine residue at codon 114 was predicted to have damaging effect on protein function by three algorithms in insilico analysis (Table 1). These findings provide evidence for the possible involvement of p.Ile114Ser mutation in disease pathogenesis.

Both the p.Met105Val and p.Met157Thr mutations have been previously identified in the Caucasian and Asian populations $[1,2,16,18,20]$. In the present study, we have identified the replacement of methionine by threonine at 157 codon position in a sporadic case (Fig. 1; family 6; I:2) with total retinal detachment in both eyes of the proband. This patient was examined at the age of 35 years with roving movements in the right eye and bilateral ectopic macula towards the temporal side. Her two sons (II:3 and II:4) were also examined and found to be clinically normal and did not harbor the observed change. In addition to this mutation, a missense change (c.694A > G; p.Met232Val) in ZNF408 of unknown significance was also detected in the proband and one of her son without any observable retinal non perfusion, indicating that the p.Met157Thr was mainly responsible for the disease phenotype in this family. The methionine at 157 codon position precedes a cysteine (codon 158), which is involved in the formation of a disulfide bond crucial for structural maintenance of CRD [39]. Based on this information, we speculate that the altered solvent interactions of hydrophilic threonine instead of hydrophobic methionine in M157T mutation may interfere with the disulfide bridge formation by following cysteine. Thus, the structural alteration caused by this mutation may interfere with the ligand interaction or FZD4 homodimer formation and could result in the pathogenicity.

The recurrent heterozygous mutation c.313A $>\mathrm{G}$ (p.Met105Val) was observed in 3 different sporadic FEVR probands (Fig. 1; families 7, 8 and 9) with advanced stages of FEVR. All the probands having this mutation developed falciform folds and tractional retinal detachment either in one or both eyes within a few months of their birth. Among the three families, two of the mothers (Families 7 and 9; I: 2) and a sibling of the proband 9 (II:1) failed to show severe retinal abnormalities despite of harboring a single copy of mutant allele. However, the mother of the proband 8 (I:2) showed peripheral retinal avascularization.
Notably, like the M157T, the M105V mutations also affected a cysteine residue at codon 106 that plays a crucial role in CRD structure formation via disulfide bridge formation with another cysteine at codon 45. Replacement of the highly conserved methionine at codon 105 with a valine residue led to severe impairment of FZD4 signaling (Fig. 2) [30]. This information strongly suggested the pathogenic nature of this missense mutation.

\section{Mutations detected in the TSPAN12 gene}

So far, 27 different mutations in TSPAN12 have been reported in FEVR patients [1, 2, 4, 5, 22-25, 59]. These mutations included missense, nonsense, small base pair deletions, insertions and splice site mutations. A novel, heterozygous c.125 $\mathrm{T}>\mathrm{C}$ (p.Val42Ala) missense mutation was identified in a female proband (IV: 2) of a consanguineous FEVR family 10 (Fig. 1). The proband developed bilateral nystagmus along with attached retrolental membranes to the posterior capsule and funnel shaped retinal folds at 4 months of age. This mutation was also observed in the maternal uncle (III: 3 ) and aunt (III: 6) of the proband who was reported to be blind since birth. However, both the parents of the proband harboring this heterozygous change were clinically normal. Another novel missense mutation c.479G > A (p.Cys160Tyr) was identified in probands of four unrelated FEVR families (Fig. 1; families 11 to 14). In two probands (IV: 2) of consanguineous FEVR families (11 and 12), this mutation was found in a homozygous condition and showed autosomal recessive inheritance. The probands of the other two FEVR families (13 and 14) were heterozygous for this change. The two probands having homozygous mutation exhibited total retinal detachments with a funnel shaped detached retinas within a few months after birth. The probands with heterozygous mutation progressively developed exudative or tractional retinal detachments with fibrovascular proliferations during the first or second decades of their life (Fig. 4). The other members of these families harboring this mutation showed variable clinical expressivity that ranged from very mild peripheral retinal non perfusion to exudation, falciform retinal folds and total retinal detachments. The three prediction tools used for insilico analysis indicated the damaging effect of this non-conservative substitution on protein function.

The third missense mutation c.334G > A (p.Val112Ile) was identified in homozygous condition in a male proband of FEVR family 15 . This patient was first examined in our hospital at 24 years of age and found to have total retinal detachment with falciform retinal folds in both eyes with bilateral nystagmus at the initial examination (Fig. 4). The mother and a male sibling of the proband, who also harbored this mutation in heterozygous state, were asymptomatic with mild pigmentary changes in the retina. This change was previously reported in 

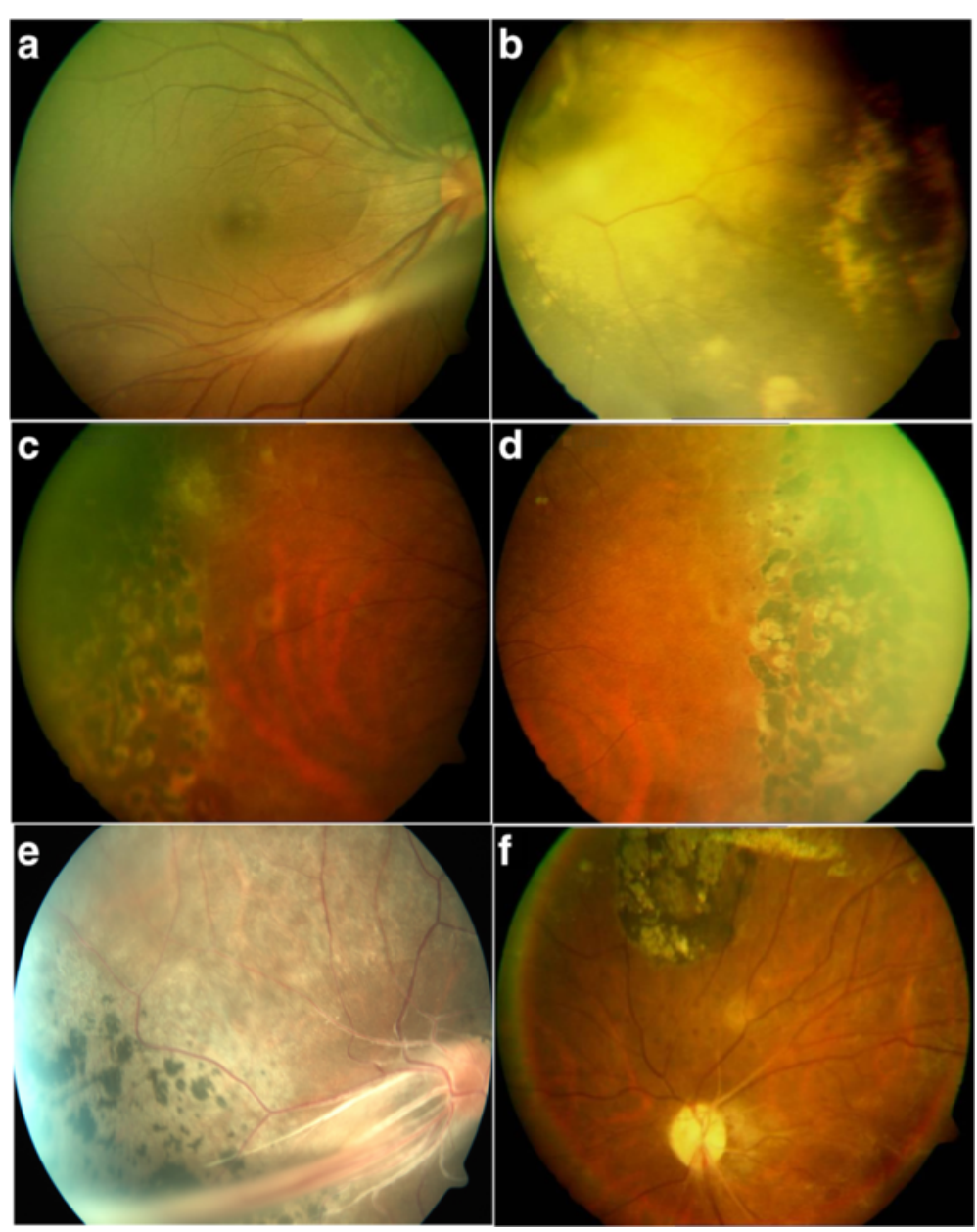

Fig. 4 Fundus photographs of the FEVR patients with novel nucleotide changes identified in the TSPAN12 gene. a\&b Patient ID: family 14-III:2 (c.479G > A); a right eye of the proband of FEVR family 14 shows slightly dragged macula. $\mathbf{b}$ left eye of the proband of FEVR family 14 shows avascular peripheral retina and exudation. c\&d Patient ID: family 14-III:4 (c.479G > A) right and left eye of a family member of FEVR family 14 shows avascular peripheral retina and scars of laser photocoagulation treatment. e\&f Patient ID: family 15-IV:2 (c.334G > A) right and left eyes of proband of FEVR family 15 shows straightening of blood vessels, retinal pigmentation, dragged macula and exudation

heterozygous condition as a rare variant by Exome Aggregation Consortium with the frequency of minor allele to be 0.0001 [47].

Another novel missense change, p.Cys160Tyr was prevalent (3.6 \% of the FEVR probands) in the present study. This mutation was observed in the probands and their affected family members of four unrelated FEVR families (Fig. 1; families 11 to 14). Two severely affected probands of the FEVR families (11 and 12) had homozygous p.Cys160Tyr mutation while the remaining probands of two FEVR families (13 and 14) and their affected relatives were heterozygous. Compared to probands with the homozygous mutations, the patients with the heterozygous allele showed a slower progression of the disease or relatively less severe ocular phenotype, suggesting the recessive inheritance pattern of the disease and effect of allelic dosage on disease severity. The previously described FEVR patients with homozygous mutations in the candidate genes had severely reduced protein expression that was shown to be responsible for severity and rapid progression of the disease [21, 23, 60].

The p.Val42Ala mutation was located in the small extracellular loop while, p.Val112Ile and p.Cys160Tyr mutations were located in the large extracellular loop of the protein. The wild type cysteine as seen for p.Cys160Tyr mutation, is located at the second position in the highly conserved CCG signature motif of extracellular loop 2 (ECL2) of the TSPAN12 protein (Fig. 2). This cysteine residue was predicted to be involved in the formation of a disulfide bridge with another cysteine residue located at the 181 position of TSPAN12. The ECL2 was suggested to be involved in protein-protein interactions with targeted proteins in the cell membrane [61]. Therefore, the disrupted structure of ECL2 due to p.Cys160Tyr 
mutation could probably interfere in TSPAN12 interaction with FZD4 protein, thereby affecting the NorrinFZD4 signaling.

\section{Mutations detected in the ZNF408 gene}

A novel, heterozygous, c.130C $>\mathrm{T}$ (p.Pro44Ser) change was observed in a male proband (II: 2) of FEVR family 16 (Fig. 5). The proband had bilateral total retinal detachments with retrolental membranes at 2 months of age. The father of the proband (I: 2) was found to be normal both phenotypically and genotypically while the mother (I: 1) remained unavailable for genetic analysis. All the three algorithims predicted it to be of benign nature. The second novel heterozygous c.2145G $>\mathrm{T}$ (p.Glu715Asp) change was observed in a sporadic proband of FEVR family 17 . The proband exhibited total retinal detachment in the right eye and subtotal retinal detachment including macula in the left eye at one year of age. The mother of the proband (I: 2) was found to be clinically normal without this variation, while the father (I: 1) was unavailable for genetic analysis. A previously reported T617N change located in tenth zinc finger domain of the protein was also observed in a family, which could potentially interfere with DNA interaction. However, the presence of this change in the unaffected mother of the proband and in a control individual is suggestive for a less or non pathogenic nature of this variation. In family 6 , the proband had M232V missense change in the ZNF408 gene and also a heterozygous M157T mutation in FZD4 gene. The heterozygous $\mathrm{M} 232 \mathrm{~V}$ change was also observed in one of the two unaffected sons of the FEVR proband (Fig. 5) however, but they lacked the M157T mutation of FZD4. The data thus suggested that M157T mutation was mainly responsible for the ocular phenotype in the proband. Further structural and functional studies are required to understand the effect of these missense changes on ZNF408 protein and its downstream interactions.

Of the three novel non-synonymous variations observed in the recently identified ZNF408 gene, the p.Pro44Ser and p.Met232Val changes were predicted to be harmless by the three algorithms used in in silico analysis and were considered as variations of unknown significance, while an evolutionarily non-conservative substitution p.Glu715Asp was predicted to be pathogenic (Fig. 2). None of these changes were seen in the controls (Table 1).

Interestingly, none of the variations in ZNF408 gene in our Indian cohort were observed in the Caucasian and Asian FEVR patients indicating the allelic heterogeneity of this condition. In summary, the present study provides an independent validation of the involvement of ZNF408 in FEVR from an ethnically diverse population from India. This also expands the mutation spectrum of ZNF408 in the pathogenesis of FEVR that would need to be further supplemented with diverse data from other populations.

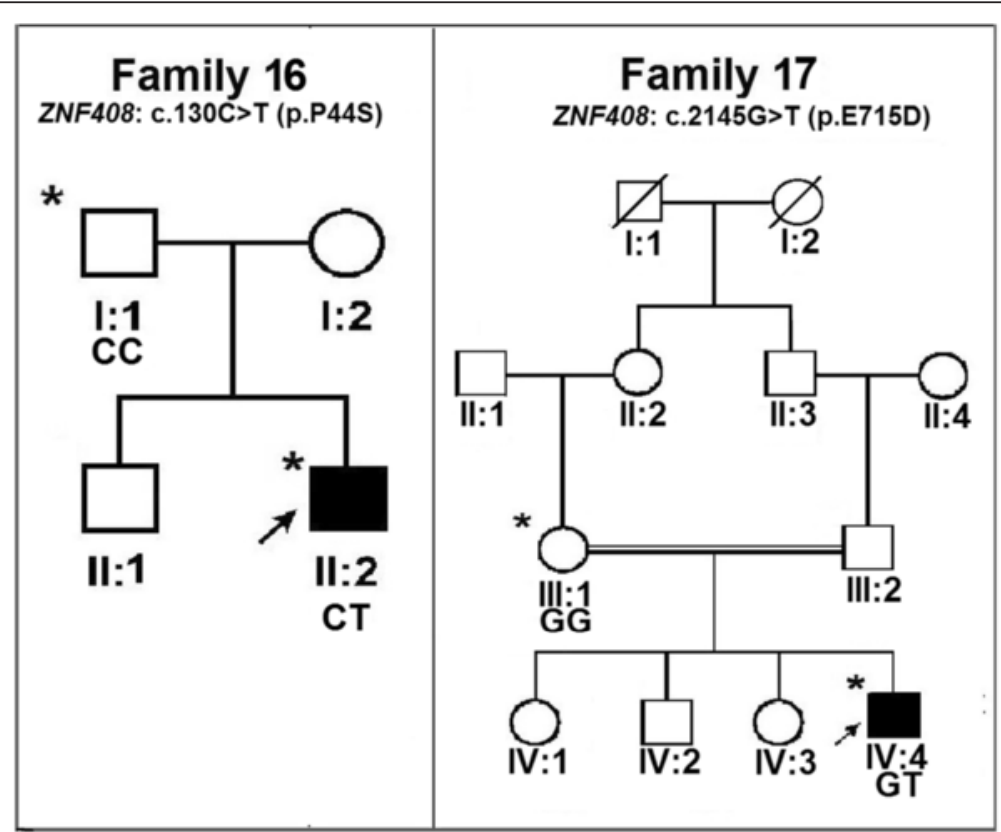

Fig. 5 Pedigrees of the FEVR families with nucleotide changes in ZNF408 gene. Completely shaded symbols represent severe stages of the disease. Open symbols represent unaffected individuals. Asterisk $\left(^{*}\right)$ over the pedigree symbols represent the individuals screened for nucleotide changes in ZNF408 gene. - indicates the presence of deletion/insertion and + indicates the wild type allele. The identified nucleotide change is represented above each pedigree 


\section{Conclusion}

In summary, the mutation frequencies of the 3 candidate genes among the Indian FEVR patients were comparable to previous reports in FZD4 (3.5-40 \%) [1, 2, 7, 8, 14-20, 42], and TSPAN12 (3.5-10\%) [1, 2, 4, 5, 22-25] genes in different populations. In previous reports the frequency of FEVR patients with ZNF408 mutations varied from 0 to $1.8 \%$ in different populations [1-3]. The frequency of patients with FZD4 mutations in our cohort was relatively higher when compared to a previous study by Nallathambi et al., in another cohort of Indian FEVR patients [42]. This indicates the broad spectrum of FZD4 mutations in FEVR pathogenesis among the Indian patients. Other than the common ocular features of FEVR, we did not recognize any distinguishable features specific to the cases harboring FZD4, TSPAN12 and ZNF408 mutations in these patients for undertaking genotype-phenotype correlation. The novel mutations identified in the present study further broadened the mutation spectrum in FEVR patients, which would facilitate better genetic counseling and diagnostics to treat these patients before the development of severe visual complications.

\section{Ethics (and consent to participate)}

The study was approved by the Institutional Review Board (IRB) of L.V. Prasad Eye Institute, Hyderabad, (Ref no LEC06104) India, and adhered to tenets of the Declaration of Helsinki. A written informed consent for research purposes was obtained from each subject prior to their participation in the study. In case of minors, the consent for participation in the study was obtained from either of the parents.

\section{Consent to publish}

Not applicable as the study does not identify the subjects and no personal information identifying the subject included in the manuscript.

\section{Availability of data and materials}

The data pertaining the study would be available from the corresponding author and principal investigator of the study upon request for any research use.

\section{Additional file}

Additional file 1: Primer and Amplification Details for the Three Candidate genes. (DOCX 17 KB)

\section{Abbreviations}

CRD: cysteine rich domain; DNA: Deoxyribose Nucleic Acid; ECL: extra cellular loops; ESP5400: Exome Sequencing Project 5400; ExAC: Exome aggregation consortium; FEVR: Familial Exudative Retinopathy; FZD4: Frizzled 4; IRB: Institutional Review Board; KIF11: Kinesin family member; LRP5: Low density lipoprotein receptor like protein 5; NDP: Norrie disease pseudoglioma; PCR: Polymerase Chain reaction; PolyPhen-2: Polymorphism Phenotyping Version
2; SIFT: Sorting Intolerant from Tolerant; TCF/LEF: T-cell factor/lymphoid enhancer factor; TSPAN12: Tetraspanin-12; ZNF408: Zinc finger prortein-408.

\section{Acknowledgments}

The authors would like to acknowledge the contribution of Dr Chitra Kannabiran for the help in initial sampling of the families affected with FEVR.

\section{Authors' contributions}

All authors conceived of and designed the experimental protocol. IK and SC contributed to the study design, analyzed the data and performed critical revision of the manuscript for important intellectual content. SJ performed the eye examinations and clinical correlations of the data. GRM and SH collected the samples and performed the experiments. GRM and IK wrote the paper. All authors have read and approved the final manuscript.

\section{Competing interests}

The authors declare that they have no competing interests.

\section{Financial support}

The study is partly supported by funding from Hyderabad Eye Research Foundation and partly by the Department of Biotechnology, Ministry of Science and Technology, Government of India (Grant \# BT/01/COE/06/02/10).

\section{Author details}

${ }^{1}$ Kallam Anji Reddy Molecular Genetics Laboratory, Brien Holden Eye Research Centre, L V Prasad Eye Institute (KAR Campus), Road\#2, Banjara Hills, Hyderabad 500034, India. ${ }^{2}$ Smt. Kanuri Santhamma Centre for Vitreo Retinal Diseases, LV Prasad Eye Institute, Hyderabad, India.

Received: 8 April 2016 Accepted: 10 May 2016

Published online: 17 June 2016

\section{References}

1. Salvo J, Lyubasyuk V, Xu M, Wang H, Wang F, Nguyen D, et al. Nextgeneration sequencing and novel variant determination in a cohort of 92 familial exudative vitreoretinopathy patients. Invest Ophthalmol Vis Sci. 2015:56:1937-46.

2. Seo SH, Yu YS, Park SW, Kim JH, Kim HK, Cho SI, et al. Molecular Characterization of FZD4, LRP5, and TSPAN12 in Familial Exudative Vitreoretinopathy. Invest Ophthalmol Vis Sci. 2015;56:5143-51.

3. Collin RW, Nikopoulos K, Dona M, Gilissen C, Hoischen A, Boonstra FN, et al. ZNF408 is mutated in familial exudative vitreoretinopathy and is crucial for the development of zebrafish retinal vasculature. Proc Natl Acad Sci U S A. 2013:110:9856-61.

4. Nikopoulos K, Gilissen C, Hoischen A, van Nouhuys CE, Boonstra FN, Blokland EA, et al. Next-generation sequencing of a $40 \mathrm{Mb}$ linkage interval reveals TSPAN12 mutations in patients with familial exudative vitreoretinopathy. Am J Hum Genet. 2010:86:240-7.

5. Poulter JA, Ali M, Gilmour DF, Rice A, Kondo H, Hayashi K, et al. Mutations in TSPAN12 cause autosomal-dominant familial exudative vitreoretinopathy. Am J Hum Genet. 2010:86:248-53.

6. Toomes C, Bottomley HM, Jackson RM, Towns KV, Scott S, Mackey DA, et al. Mutations in LRP5 or FZD4 underlie the common familial exudative vitreoretinopathy locus on chromosome 11q. Am J Hum Genet. 2004;74: 721-30.

7. Toomes C, Bottomley HM, Scott S, Mackey DA, Craig JE, Appukuttan B, et al. Spectrum and frequency of FZD4 mutations in familial exudative vitreoretinopathy. Invest Ophthalmol Vis Sci. 2004;45:2083-90.

8. Robitaille J, MacDonald ML, Kaykas A, Sheldahl LC, Zeisler J, Dube MP, et al. Mutant frizzled-4 disrupts retinal angiogenesis in familial exudative vitreoretinopathy. Nat Genet. 2002;32:326-30.

9. Shastry BS, Hejtmancik JF, Trese MT. Identification of novel missense mutations in the Norrie disease gene associated with one X-linked and four sporadic cases of familial exudative vitreoretinopathy. Hum Mutat. 1997;9: 396-401.

10. Chen ZY, Battinelli EM, Fielder A, Bundey S, Sims K, Breakefield XO, et al. A mutation in the Norrie disease gene (NDP) associated with X-linked familial exudative vitreoretinopathy. Nat Genet. 1993;5:180-3.

11. Shukla D, Singh J, Sudheer G, Soman M, John RK, Ramasamy K, et al. Familial exudative vitreoretinopathy (FEVR). Clinical profile and management. Indian J Ophthalmol. 2003;51:323-8. 
12. Pendergast SD, Trese MT. Familial exudative vitreoretinopathy. Results of surgical management. Ophthalmology. 1998;105:1015-23.

13. Gow J, Oliver GL. Familial exudative vitreoretinopathy. An expanded view. Arch Ophthalmol. 1971:86:150-5.

14. Boonstra FN, van Nouhuys CE, Schuil J, de Wijs IJ, van der Donk KP, Nikopoulos K, et al. Clinical and molecular evaluation of probands and family members with familial exudative vitreoretinopathy. Invest Ophthalmol Vis Sci. 2009;50:4379-85.

15. Nikopoulos K, Venselaar H, Collin RW, Riveiro-Alvarez R, Boonstra FN Hooymans JM, et al. Overview of the mutation spectrum in familial exudative vitreoretinopathy and Norrie disease with identification of 21 novel variants in FZD4, LRP5, and NDP. Hum Mutat. 2010;31:656-66.

16. Yang H, Li S, Xiao X, Wang P, Guo X, Zhang Q. Identification of FZD4 and LRP5 mutations in 11 of 49 families with familial exudative vitreoretinopathy. Mol Vis. 2012;18:2438-46.

17. Zhang K, Harada Y, Wei X, Shukla D, Rajendran A, Tawansy K, et al. An essential role of the cysteine-rich domain of FZD4 in Norrin/Wnt signaling and familial exudative vitreoretinopathy. J Biol Chem. 2011;286:10210-5.

18. Jia LY, Li XX, Yu WZ, Zeng WT, Liang C. Novel frizzled-4 gene mutations in chinese patients with familial exudative vitreoretinopathy. Arch Ophthalmol. 2010;128:1341-9.

19. Qin M, Hayashi H, Oshima K, Tahira T, Hayashi K, Kondo H. Complexity of the genotype-phenotype correlation in familial exudative vitreoretinopathy with mutations in the LRP5 and/or FZD4 genes. Hum Mutat. 2005;26:104-12.

20. Kondo H, Hayashi H, Oshima K, Tahira T, Hayashi K. Frizzled 4 gene (FZD4) mutations in patients with familial exudative vitreoretinopathy with variable expressivity. Br J Ophthalmol. 2003:87:1291-5.

21. Jiao X, Ventruto V, Trese MT, Shastry BS, Hejtmancik JF. Autosomal recessive familial exudative vitreoretinopathy is associated with mutations in LRP5. Am J Hum Genet. 2004;75:878-84.

22. Kondo H, Kusaka S, Yoshinaga A, Uchio E, Tawara A, Hayashi K, et al. Mutations in the TSPAN12 gene in Japanese patients with familial exudative vitreoretinopathy. Am J Ophthalmol. 2011;151:1095-100. e1.

23. Poulter JA, Davidson AE, Ali M, Gilmour DF, Parry DA, Mintz-Hittner HA, et al. Recessive mutations in TSPAN12 cause retinal dysplasia and severe familial exudative vitreoretinopathy (FEVR). Invest Ophthalmol Vis Sci. 2012; 53:2873-9.

24. Yang H, Xiao X, Li S, Mai G, Zhang Q. Novel TSPAN12 mutations in patients with familial exudative vitreoretinopathy and their associated phenotypes. Mol Vis. 2011;17:1128-35

25. Xu Y, Huang L, Li J, Zhang Q, Fei P, Zhu X, et al. Novel mutations in the TSPAN12 gene in Chinese patients with familial exudative vitreoretinopathy. Mol Vis. 2014;20:1296-306.

26. Robitaille JM, Gillett RM, LeBlanc MA, Gaston D, Nightingale M, Mackley MP, et al. Phenotypic overlap between familial exudative vitreoretinopathy and microcephaly, lymphedema, and chorioretinal dysplasia caused by KIF11 mutations. JAMA Ophthalmol. 2014;132:1393-9.

27. Hu H, Xiao X, Li S, Jia X, Guo X, Zhang Q. KIF11 mutations are a common cause of autosomal dominant familial exudative vitreoretinopathy. $\mathrm{Br} J$ Ophthalmol. 2016;100:278-83.

28. Junge HJ, Yang S, Burton JB, Paes K, Shu X, French DM, et al. TSPAN12 regulates retinal vascular development by promoting Norrin- but not Wntinduced FZD4/beta-catenin signaling. Cell. 2009;139:299-311.

29. Wang Y, Rattner A, Zhou Y, Williams J, Smallwood PM, Nathans J. Norrin/ Frizzled4 signaling in retinal vascular development and blood brain barrier plasticity. Cell. 2012;151:1332-44.

30. Xu Q, Wang Y, Dabdoub A, Smallwood PM, Williams J, Woods C, et al. Vascular development in the retina and inner ear: control by Norrin and Frizzled-4, a high-affinity ligand-receptor pair. Cell. 2004;116:883-95.

31. Ye X, Wang Y, Cahill H, Yu M, Badea TC, Smallwood PM, et al. Norrin, frizzled-4, and Lrp5 signaling in endothelial cells controls a genetic program for retinal vascularization. Cell. 2009;139:285-98.

32. Ye $X$, Wang $Y$, Nathans J. The Norrin/Frizzled4 signaling pathway in retinal vascular development and disease. Trends Mol Med. 2010;16:417-25.

33. Gong Y, Slee RB, Fukai N, Rawadi G, Roman-Roman S, Reginato AM, et al. $L D L$ receptor-related protein $5(L R P 5)$ affects bone accrual and eye development. Cell. 2001;107:513-23.

34. Luhmann UF, Lin J, Acar N, Lammel S, Feil S, Grimm C, et al. Role of the Norrie disease pseudoglioma gene in sprouting angiogenesis during development of the retinal vasculature. Invest Ophthalmol Vis Sci. 2005;46:3372-82.
35. Richter M, Gottanka J, May CA, Welge-Lussen U, Berger W, Lutjen-Drecoll E. Retinal vasculature changes in Norrie disease mice. Invest Ophthalmol Vis Sci. 1998:39:2450-7.

36. Xia CH, Liu H, Cheung D, Wang M, Cheng C, Du X, et al. A model for familial exudative vitreoretinopathy caused by LPR5 mutations. Hum Mol Genet. 2008;17:1605-12.

37. Xia CH, Yablonka-Reuveni Z, Gong X. LRP5 is required for vascular development in deeper layers of the retina. PLoS One. 2010;5:e11676.

38. Kirikoshi H, Sagara N, Koike J, Tanaka K, Sekihara H, Hirai M, et al. Molecular cloning and characterization of human Frizzled-4 on chromosome 11q14q21. Biochem Biophys Res Commun. 1999;264:955-61.

39. Schulte G. International Union of Basic and Clinical Pharmacology. LXXX. The class Frizzled receptors. Pharmacol Rev. 2010;62:632-67.

40. Ke J, Harikumar KG, Erice C, Chen C, Gu X, Wang L, et al. Structure and function of Norrin in assembly and activation of a Frizzled 4-Lrp5/6 complex. Genes Dev. 2013;27:2305-19.

41. Smallwood PM, Williams J, Xu Q, Leahy DJ, Nathans J. Mutational analysis of Norrin-Frizzled4 recognition. J Biol Chem. 2007;282:4057-68.

42. Nallathambi J, Shukla D, Rajendran A, Namperumalsamy P, Muthulakshmi R, Sundaresan P. Identification of novel FZD4 mutations in Indian patients with familial exudative vitreoretinopathy. Mol Vis. 2006;12:1086-92.

43. Sambrook J, Fritsch EF, Maniatis T. Molecular Cloning: A Laboratory Manual: Cold Spring Harbor laboratory Press; 1989

44. Sherry ST, Ward MH, Kholodov M, Baker J, Phan L, Smigielski EM, et al. dbSNP. the NCBI database of genetic variation. Nucleic Acids Res. 2001;29:308-11.

45. Exome Variant Server NGESP, (ESP). Seattle W, USA: eEAO. Available at: http://evs.gs.washington. 2013.

46. NIEHS Environmental Genome Project. Seattle W, USA: Ac- Aahegwen, 2013.

47. Exome Aggregation Consortium (ExAC) C, MA. Available at http://exac. broadinstitute.org. Accessed April 2015.

48. Kumar P, Henikoff S, Ng PC. Predicting the effects of coding nonsynonymous variants on protein function using the SIFT algorithm. Nat Protoc. 2009;4:1073-81.

49. Adzhubei I, Jordan DM, Sunyaev SR. Predicting functional effect of human missense mutations using PolyPhen-2. Curr Protoc Hum Genet 2013; Chapter 7:Unit7 20.

50. Schwarz JM, Rodelsperger C, Schuelke M, Seelow D. MutationTaster evaluates disease-causing potential of sequence alterations. Nat Methods. 2010;7:575-6.

51. Robitaille JM, Zheng B, Wallace K, Beis MJ, Tatlidil C, Yang J, et al. The role of Frizzled-4 mutations in familial exudative vitreoretinopathy and Coats disease. Br J Ophthalmol. 2011:95:574-9.

52. Muller M, Kusserow $C$, Orth U, Klar-Dissars U, Laqua H, Gal A. Mutations of the frizzled-4 gene. Their impact on medical care of patients with autosomal dominant exudative vitreoretinopathy. Ophthalmologe. 2008;105:262-8.

53. Robitaille JM, Wallace $K$, Zheng B, Beis MJ, Samuels M, Hoskin-Mott A, et al. Phenotypic overlap of familial exudative vitreoretinopathy (FEVR) with persistent fetal vasculature (PFV) caused by FZD4 mutations in two distinct pedigrees. Ophthalmic Genet. 2009;30:23-30.

54. Omoto S, Hayashi T, Kitahara K, Takeuchi T, Ueoka Y. Autosomal dominant familial exudative vitreoretinopathy in two Japanese families with FZD4 mutations (H69Y and C181R). Ophthalmic Genet. 2004:25:81-90.

55. Yoshida S, Arita R, Yoshida A, Tada H, Emori A, Noda Y, et al. Novel mutation in FZD4 gene in a Japanese pedigree with familial exudative vitreoretinopathy. Am J Ophthalmol. 2004;138:670-1.

56. Hentze MW, Kulozik AE. A perfect message: RNA surveillance and nonsensemediated decay. Cell. 1999:96:307-10.

57. Kaykas A, Yang-Snyder J, Heroux M, Shah KV, Bouvier M, Moon RT. Mutant Frizzled 4 associated with vitreoretinopathy traps wild-type Frizzled in the endoplasmic reticulum by oligomerization. Nat Cell Biol. 2004;6:52-8.

58. Lemma V, D'Agostino M, Caporaso MG, Mallardo M, Oliviero G, Stornaiuolo $\mathrm{M}$, et al. A disorder-to-order structural transition in the $\mathrm{COOH}$-tail of Fz4 determines misfolding of the L501fsX533-Fz4 mutant. Sci Rep. 2013;3:2659.

59. Gal M, Levanon EY, Hujeirat Y, Khayat M, Pe'er J, Shalev S. Novel mutation in TSPAN12 leads to autosomal recessive inheritance of congenital vitreoretinal disease with intra-familial phenotypic variability. Am J Med Genet A. 2014;164A:2996-3002.

60. Kondo H, Qin M, Tahira T, Uchio E, Hayashi K. Severe form of familial exudative vitreoretinopathy caused by homozygous R417Q mutation in frizzled-4 gene. Ophthalmic Genet. 2007;28:220-3.

61. Boucheix C, Rubinstein E. Tetraspanins. Cell Mol Life Sci. 2001;58:1189-205. 